\title{
Der augenblickliche Stand der dogmenhistorischen Lehre an deutschsprachigen Universitäten (1976- 1995): Fortfuhrung einer Erhebung
}

Citation for published version (APA):

Backhaus, J. G., \& Jeserich, N. (1998). Der augenblickliche Stand der dogmenhistorischen Lehre an deutschsprachigen Universitäten (1976-1995): Fortfuhrung einer Erhebung. METEOR, Maastricht University School of Business and Economics. METEOR Research Memorandum No. 004 https://doi.org/10.26481/umamet.1998004

Document status and date:

Published: 01/01/1998

DOI:

10.26481/umamet.1998004

Document Version:

Publisher's PDF, also known as Version of record

Please check the document version of this publication:

- A submitted manuscript is the version of the article upon submission and before peer-review. There can be important differences between the submitted version and the official published version of record.

People interested in the research are advised to contact the author for the final version of the publication, or visit the DOI to the publisher's website.

- The final author version and the galley proof are versions of the publication after peer review.

- The final published version features the final layout of the paper including the volume, issue and page numbers.

Link to publication

\footnotetext{
General rights rights.

- You may freely distribute the URL identifying the publication in the public portal. please follow below link for the End User Agreement:

www.umlib.nl/taverne-license

Take down policy

If you believe that this document breaches copyright please contact us at:

repository@maastrichtuniversity.nl

providing details and we will investigate your claim.
}

Copyright and moral rights for the publications made accessible in the public portal are retained by the authors and/or other copyright owners and it is a condition of accessing publications that users recognise and abide by the legal requirements associated with these

- Users may download and print one copy of any publication from the public portal for the purpose of private study or research.

- You may not further distribute the material or use it for any profit-making activity or commercial gain

If the publication is distributed under the terms of Article 25fa of the Dutch Copyright Act, indicated by the "Taverne" license above, 
den 27. Februar 1997

Der augenblickliche Stand der dogmenhistorischen Lehre an deutschsprachigen Universitäten (1976-1995): Fortführung einer Erhebung ${ }^{1}$

\author{
Jürgen Backhaus und Nadine Jeserich \\ Maastricht University, AE \\ Postfach 616 \\ 6200 MD Maastricht \\ die Niederlande \\ tel: $+31-43-3883652 / 3636$ \\ fax: $+31-43-3258440$ \\ email: f.schijlen@algec.unimaas.nl
}

J.E.L. code: A20, B10

$1 \quad$ Vgl. Backhaus (1982) 


\begin{abstract}
This note represents results about the extent of teaching the history of economic thought at German language based universities. At the time when cuts need to be made, many universities are thought of cutting non-essential courses first, of which the history of economic thought might be considered to be a likely candidate. Not so. Only roughly four per cent of our responding faculties have made this choice, the others are continuing their previous practice even despite the severe cutbacks and increasing teaching loads they have experienced during the survey period.
\end{abstract}




\section{Der augenblickliche Stand der dogmenhistorischen Lehre an deutschsprachigen Univer- sitäten (1976-1995): Fortführung einer Erhebung}

Die Studie hatte das Ziel, die Entwicklung der theoriegeschichtlichen wirtschaftswissenschaftlichen Lehrveranstaltungen an deutschsprachigen Hochschulen zu dokumentieren. Es handelt sich um eine Vollerhebung in dem Sinne, daß alle Hochschulen, die zum Erhebungszeitpunkt über wirtschaftswissenschaftliche Studiengänge verfügten, in die Untersuchung einbezogen wurden. Die Erhebungsunterlagen enthielten einen Erhebungsbogen, der fragte, ob theoriegeschichtliche Lehrveranstaltungen seit dem Sommersemester 1976 stattgefunden hätten, mit wievielen Wochenstunden sie dotiert waren, wie der Name des Veranstalters und die Bezeichnung der Veranstaltung lautete, und in welchem Sinne die Veranstaltung(en) prüfungsrelevant war(en).

Die Unterlagen wurden Ende Juni 1996 an die betreffenden Hochschulen versandt und am 20.01.1997 endgültig ausgewertet.

Von den insgesamt 80 angeschriebenen Hochschulen (65 in Deutschland, 8 in Österreich, 6 in der Schweiz und eine in Italien) antworteten 74, was einer Rücklaufquote von 92\% entspricht. Von den 74 Fakultäten, die geantwortet hatten, konnten 55 positiv über theoriegeschichtliche Lehrveranstaltungen berichten, also rund 74\%. Unter diesen befanden sich vier Universitäten, die darauf hinwiesen, sie integrierten theoriegeschichtliche Themen in andere Lehrveranstaltungen. ${ }^{2}$ Vier weitere Universitäten teilten mit, sie erwögen Aspekte der Theoriegeschichte vermehrt zu betonen, bzw. planten eine Einrichtung eines Wahlfaches Wirtschaftsgeschichte einschließlich Dogmengeschichte.

Zwischen 1976 und 1996 waren theoriegeschichtliche Lehrveranstaltungen an den deutschsprachigen Hochschulen keine Ausnahme. Wir haben nicht weniger als 1064 Lehrveranstaltungen ermittelt, mit insgesamt 2516 Wochenstunden.

Die Lehrveranstaltungen sind überwiegend in Vorlesungsform abgehalten worden. Es gibt aber Ausnahmen. Zum Beispiel theoriegeschichtliche "Übungen", Schwerpunktseminare und Kolloquien. Die Erhebungen lassen keine genauen Rückschlüsse darauf zu, zu welchem Zeitpunkt im Studium die Veranstaltungen stattfinden. Einzelne Fakultäten bieten Theoriegeschichte als Einführungsvorlesung oder im Grundstudium an, während sie an anderen Universitäten den Studenten im Haupstudium vorbehalten sind.

Die Lehrveranstaltungen gelten fast im gleichen Maße als Haupt- und Nebenfach und werden zum größten Teil obligatorisch verlangt. Einige Universitäten gaben nur an, daß die Veranstaltungen obligatorisch seien, an anderen Fakultäten sind sie in unterschiedlichen Abstufungen (zum Beispiel Wahlplichtfach) fakultativ.

2 Dies geschieht nicht nur an diesen vier Universitäten, sondern vielerorts. Insofern wird mit unserer Erhebung das Ausmaß der dogmenhistorischen Lehre an deutschsprachigen Universitäten systematisch unterschätzt.

Vgl. hierzu explizit Krelle, 1997. In seiner Vorlesung über Konjunkturtheorie etwa geht Krelle an der Universität Bonn auf die Theorien Wicksells, Mises, Röpkes, Spiethoffs, Schumpeters, Jöhrs und Schmölders ein, um nur einige zu nennen. Die

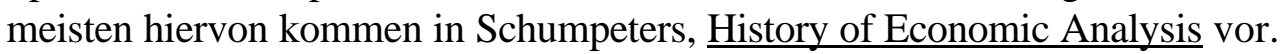


Regelmäßigkeit und Kontinuität der Lehrveranstaltungen sind offenbar weitgehend eine Funktion der personellen Zusammensetztung der Fakultäten. Lücken traten überwiegend dann auf, wenn Professoren emeritiert wurden oder die Hochschule wechselten. Es bieten aber immerhin achtzehn Hochschulen seit mindestens zehn Jahren regelmäßig theoriegeschichtliche Lehrveranstaltungen an, fünf sogar kontinuierlich seit 1976. Drei weitere Hochschulen berichten allerdings, daß sie diese im folgenden Semester aufgrund ihrer personellen Situation, bzw. fehlendem Interesse der Studenten nicht mehr anbieten werden.

Die Inhalte der Lehrveranstaltungen haben wir versucht, aus den ausgewiesenen Titeln zu erschließen wo nähere Beschreibungen nicht vorhanden waren. Diese Methode läßt aber nur eine Grobaufteilung zu.

Die meisten Lehrveranstaltungen waren als allgemeiner Überblick angelegt. Es wurden insgesamt 497 ermittelt. Diese Kategorie war gefolgt von 164, die sich besonderer Fragestellungen in dogmengeschichtlicher Sicht widmeten, 134 Lehrveranstaltungen, die einzeldisziplinäre Ansätze behandelten, und 143 Lehrveranstaltungen stuften wir als abschnitts- oder epochenbezogen ein und 98 Lehrveranstaltungen rückten eine bestimmte Person in den Mittelpunkt der Betrachtung.

Am häufigsten innerhalb der Kategorie der allgemeinen Lehrveranstaltungen tauchten die Kurstitel "Dogmengeschichte" und "Geschichte der volkswirtschaftlichen Lehrmeinungen bzw. der Volkswirtschaftslehre" auf. Es finden sich ferner die Bezeichnungen "Wissenschaftsgeschichte", "Theoriegeschichte" und "Geschichte der Wirtschaftstheorie".

Unter den besonderen Veranstaltungen zur Dogmengeschichte fassen wir personenbezogene Ansätze, epochenbezogene Ansätze, auf Einzeldisziplinen bezogene Ansätze und die Verfolgung besonderer Fragestellungen zusammen.

Karl Marx und J.M. Keynes sind mit weitem Abstand die beliebtesten Themen im Rahmen personenbezogener Ansätze. Weiterhin werden Vorlesungen über J.A. Schumpeter, A. Smith, W. Sombart, A. Marshall, J.S. Mill und M. Weber erwähnt.

In der Regel handelt es sich bei den abschnitts- und epochenbezogenen Veranstaltungen um den Versuch, die gesamte dogmengeschichtliche Vorlesung in einzelne, chronologisch vorgegebene Abschnitte einzuteilen. Es finden sich aber auch andere Beispiele, wie etwa "Allgemeine Wirtschaftsgeschichte des 19. und 20. Jahrhunderts", "Einführung in die neoklassische Theorie" oder "Vom Merkantilismus zur klassischen Nationalökonomie".

Die Kurstitel zu einzeldisziplinären Ansätzen zeigen, daß oft besondere Fragestellungen verfolgt wurden, zum Beispiel "BWL-Grundlagen Wirtschaftsgeschichte", "Einführung in die Unternehmensgeschichte", "Geschichte der Geldtheorie", "Geschichte der Wachstums- und Entwicklungstheorie" oder "Geschichte wirtschaftspolitischer Konzeptionen".

Es zeigte sich auch, daß sehr unterschiedliche Fragestellungen theoriegeschichtlich angegangen werden können, etwa "Gesellschaftliche Arbeit und ökonomische Theorie", "Beschäftigung und Arbeitswelt im 19. und 20. Jahrhundert", "Politische Ökonomie" oder ein Seminar "Macht und ökonomisches Gesetz".

Die Mitarbeit an der Umfrage lag überwiegend bei Dozenten, die die dogmengeschichtliche wirtschaftswissenschaftliche Lehrveranstaltung an ihrer Hochschule anboten oder angeboten haben. Es antwortete aber auch immerhin in sieben Fällen der Dekan persönlich.

\section{Literatur:}

Jürgen Backhaus, Theoriegeschichte - Wozu? Eine theoretische und empirische Untersuchung 
In: Fritz Neumark (Hrsg.), Studien zur Entwicklung der ökonomischen Theorie III. Berlin: Duncker \& Humblot 1982 (Schriften des Vereins für Socialpolitik N.F. 115/III), pp. 139-167.

Wilhelm Krelle, History of Economic Thought: Is it an Important Ingredient of Teaching Economics?, paper read at Maastricht University, 20 February 1997.

Joseph Alouis Schumpeter, History of Economic Analysis, New York: Oxford University Press, 1954. 